\title{
PHOTOMETRIC INVESTIGATION OF IC 2944
}

\author{
A. ARDEBERG, E. MAURICE, and J. RICKARD \\ European Southern Observatory, Santiago, Chile
}

\begin{abstract}
An investigation has been made of the central region of the cluster IC 2944. For about 70 stars $U B V$ photometry has been made. For the brighter of those stars also $\mathrm{H} \beta$ has been measured. Slit spectra of intermediate dispersion $\left(73 \AA \mathrm{mm}^{-1}\right)$ have been made for the 40 brightest stars. Coudé spectra $\left(12 \AA \mathrm{mm}^{-1}\right)$ have been obtained for 8 stars. Fabry-Pérot measurements have been made in 5 points within the nebulosity. The number of blue stars is shown to be extremely high. Down to visual magnitude 11.5 the cluster seems to contain only $\mathrm{O}$ and $\mathrm{B}$ stars, the majority of spectral class earlier than B3. The Fabry-Pérot measurements give evidence of high internal gas motion. Evidently the gas is thin, visible only because of the great number of hot stars.

Details will be published elsewhere.
\end{abstract}

